Ergod. Th. \& Dynam. Sys. (1982), 2, 1-13

Printed in Great Britain

\title{
Iterated extensions
}

\author{
ROBERT ELLIS AND SHMUEL GLASNER
}

\author{
Minnesota 55455 and \\ Department of Mathematics, Tel Aviv University, Tel Aviv, Israel
}

(Received 21 May 1981 and revised 22 March 1982)

Abstract. The notion of an iterated extension of a flow is introduced and studied. In particular it is shown how eigenfunctions occur in a natural way. This is then exploited to produce an example of a weakly mixing minimal set with a non-iveakly mixing quasi-factor.

\section{Introduction}

The flow $(Y, T)$ is an extension of the flow $(X, T)$ if there exists an epimorphism of $(Y, T)$ onto $(X, T)$. One way of producing extensions of $(X, T)$ is by means of cocycles of $(X, T)$ into a compact group $K$. Thus let $\sigma$ be a cocycle on $(X, T)$ to $K$. Then one forms the skew product flow $\left(K \times_{\sigma} X, T\right)$ where the action of $T$ on $K \times X$ is given by the map

$$
(k, x, t) \mapsto(k \sigma(x, t), x t): K \times X \times T \rightarrow K \times X .
$$

When the phase group $T$ is isomorphic to the integers, the set of cocycles on $(X, T)$ to $K$ may be identified with the set of continuous functions on $X$ to $K$ and this may be exploited to iterate the extension.

In order to illustrate the basic definitions and facilitate the reading of the paper we describe this construction informally.

A flow in this paper is a pair $(X, t)$ consisting of a compact Hausdorff space $X$ and a homeomorphism $t$ of $X$ onto itself. (We use the same letter, $t$, for every flow considered.) $K$ will stand for a compact abelian topological group, $e$ the identity element of $K$, and $Z(X ; K)$ the set of 1-cocycles from $X \times \mathbb{Z}$ into $K$.

Now start with a minimal pointed flow $\left(X, x_{0}\right)$ and a cocycle $\sigma \in Z(X ; K)$. Let $\left(Y, y_{0}\right)$ be the pointed flow ext $(X, \sigma)$. Thus $Y \subset K \times X$ is the orbit closure of $y_{0}=\left(e, x_{0}\right)$ in the flow on $K \times X$ given by

$$
(k, x) t=(k \sigma(x, t) x, t) \text {. }
$$

We let $F_{\sigma}: X \rightarrow K$ be the function on $X$ defined by $F_{\sigma}(x)=\sigma(x, t)$. Next we consider the function

$$
\delta \sigma(k, x)^{-1} \delta \sigma\left((k, x) t^{n}\right)=\sigma\left(x, t^{n}\right),
$$

$\delta \sigma$ is the function on $Y$ which 'co-bounds' $\sigma$. Our next step is to define a cocycle on $Y$ by means of the function $\delta \sigma$. Namely let $\sigma_{1} \in Z(Y, K)$ be given by

$$
\sigma_{1}(y, t)=F_{\sigma_{1}}(y)=\delta \sigma(y) \text {. }
$$


Let $Y_{1}=\operatorname{ext}\left(Y, \sigma_{1}\right) \subset K \times K \times X$; i.e. $Y_{1}$ is the orbit closure of $z_{0}=\left(e, e, x_{0}\right)$ in the flow on $K \times K \times X$ given by

$$
(l, k, x) t=\left(l F_{\sigma_{1}}(k, x), k F_{\sigma}(x), x t\right) .
$$

(Writing down the orbit of $z_{0}$ in $Y_{1}$ we have

$$
\left.\left(e, e, x_{0}\right) t^{n}=\left(\sigma_{1}\left(y_{0}, t^{n}\right), \sigma\left(x_{0}, t^{n}\right), x_{0} t^{n}\right)=\left(\delta \sigma_{1}\left(z_{0} t^{n}\right), \delta \sigma\left(y_{0} t^{n}\right), x_{0} t^{n}\right) .\right)
$$

The function $\delta \sigma_{1}: Y_{1} \rightarrow K, \delta \sigma_{1}(l, k, x)=l$ can now be used to define $\sigma_{2} \in Z\left(Y_{1} ; K\right)$ namely

$$
\sigma_{2}(z, t)=F_{\sigma_{2}}(z)=\delta \sigma_{1}(z) \text { etc. }
$$

We were motivated to study the structure of these extensions by our attempts to understand why our construction of eigenfunctions from cocycles in [4] 'worked'. This is explained in proposition 1.3 which states that for a proper choice of $\alpha$ an eigenfunction will appear in the supremum of the flows ext $(x, \sigma)$ and ext $(x, \sigma) \alpha$.

As an added bonus our analysis allowed us to construct a weakly mixing flow with a non-weakly mixing quasi-factor. (Recall that a quasi-factor of a flow $Z$ is a minimal subset of the flow induced on $2^{Z}$, the space of closed subsets of $Z$.) To see how this is done suppose that in the procedure described above we take $X$ to be weakly mixing, $K=\mathbb{K}$ the circle group and choose $\sigma$ such that $Y=\mathbb{K} \times X$ and $Y$ is also weakly mixing (this is possible by [6]). We then show that necessarily $Y_{1}=\mathbb{K} \times \mathbb{K} \times X$ (proposition 1.7) and that $Y_{1}$ is weakly mixing (corollary 1.11). Take $\mathscr{Y}$ to be the orbit closure of say

$$
\left\{\left(1,-1, x_{0}\right),\left(1,1, x_{0}\right)\right\} \text {, }
$$

in $2^{Y_{1}}$, the flow of closed subsets of $Y_{1}$. It is easy to see that the minimal flow 9 has -1 as an eigenvalue, in particular it is not weakly mixing. We then show that $y=\mathfrak{a}(A, Y)$ for a certain $\tau$-closed subgroup $A$ of $G$.

These subjects together with the analysis of flows of the form

$$
\left(Y, y_{0}\right) \vee\left(Y, y_{1}\right) \text {, }
$$

where $y_{0}$ and $y_{1}$ project onto the same point in $X$, on which our results rest, are the content of $\S 1$. In $\S 2$ we consider the higher order cocycles $\sigma_{n}(n \in \mathbb{N})$ and generalize some of the results of $\S 1$.

We now formalize the above definitions. Each flow $(X, t)$ will be assumed provided with a base point $x_{0}$ such that $x_{0} u=x_{0}$, where $u$ is a fixed idempotent in some minimal subset $M$ of $\beta \mathbb{Z}$. This allows us to pass back and forth between minimal sets and $\mathbb{Z}$-subalgebras of $C(M)$. The algebra corresponding to $X$ will be denoted by al $(X)$ and the flow corresponding to the algebra $\mathscr{A}$ by $|\mathcal{A}|$. (See [1] for details.)

Let $K$ be a compact abelian topological group. Then $Z(M, K)$ will denote the set of cocycles on $M$ to $K$ and

$$
Z(X, K)=\{\sigma \in Z(M, K): \text { al }(\sigma) \subset \text { al }(X)\} .
$$

There is a bijective correspondence

$$
\sigma \leftrightarrow F_{\sigma}: Z(X, K) \leftrightarrow C(X, K),
$$

the set of continuous functions from $X$ to $K$, given by

$$
F_{\sigma}(x)=\sigma(x, t) \text {. }
$$


On the other hand there is a bijective correspondence

$$
\sigma \rightarrow \delta \sigma: Z(M, K) \rightarrow C_{0}(M, K)=\{f \in C(M, K): f(u)=e\}
$$

(e, the identity element of $K)$. Consequently given $\sigma \in Z(M, K)$ there exists $\partial_{\sigma} \in Z(M, K)$ such that

$$
\delta \partial_{\sigma}(m)=F_{\sigma}(u)^{-1} F_{\sigma}(m) \quad(m \in M) .
$$

(For a detailed discussion of the correspondence $\sigma \rightarrow \delta \sigma$ see [2]. In the latter $\delta \sigma$ is denoted by $f_{\sigma \cdot}$ )

We write $\mathbb{R}$ for the real numbers, $\mathbb{Z}$ for the integers, and $\mathbb{K}$ for the multiplicative circle group.

\section{Section 1}

The first result of this section codifies the relationship among the various operations on a cocycle described above. Since the proof follows directly from the definitions, it will be omitted.

(1.1) Proposition. Let $\sigma \in Z(M, K)$. Then:

(1) $\left(\delta \partial_{\sigma}\right)(m)=F_{\sigma}(u)^{-1} F_{\sigma}(m)$.

(2) $\sigma(m, t)=F_{\sigma}(u)\left(\delta \partial_{\sigma}\right)(m)$.

(3) $F_{\sigma}(u)^{-1} \delta \sigma(m, t)=\delta \partial_{\sigma}(m) \delta \sigma(m)$.

(4) $\left(\delta \partial_{\sigma}\right)(m)\left(\delta \partial_{\sigma}\right)(n)^{-1}=F_{\sigma}(m) F_{\sigma}(n)^{-1}(m, n \in M)$.

Notice that (2) implies that if $\sigma$ is a cocycle on $X$ to $K$, then $\partial_{\sigma}$ is a coboundary on $X$ to $K$.

(1.2) Proposition. Let $\sigma \in Z(M, K), m \in M$, and $\omega$ be that element of $Z(M, K)$ with

$$
\delta \omega(x)=\delta \sigma(x)^{-1} \delta \sigma(m)^{-1} \delta \sigma(m x) \quad(x \in M) .
$$

Then $F_{\omega}(x)=\delta \partial_{\sigma}(m x) \delta \partial_{\sigma}(x)^{-1} \quad(x \in M)$.

Proof.

$$
\begin{aligned}
F_{\omega}(x) & =\omega(x, t)=\delta \omega(x)^{-1} \delta \omega(x t) \\
& =\delta \sigma(m x)^{-1} \delta \sigma(m) \delta \sigma(x) \delta \sigma(x t)^{-1} \delta \sigma(m)^{-1} \delta \sigma(m x t) \\
& =\delta \sigma(m x)^{-1} \delta \sigma(x) \delta \sigma(x t)^{-1} \delta \sigma(m x t) \\
& =\sigma(x, t)^{-1} \sigma(m x, t)=F_{\sigma}(x)^{-1} F_{\sigma}(m x) \\
& =\delta \partial_{\sigma}(x)^{-1} \delta \partial_{\sigma}(m x) .
\end{aligned}
$$

(1.3) Proposition. Let $\sigma \in Z(A, K), \alpha \in \mathrm{g}\left(\partial_{\sigma}\right)$ and $F_{\omega}(m)=\delta \partial_{\sigma}(\alpha)(m \in M)$. Then

(1) al $(\delta \omega) \subset \operatorname{ext}(\mathscr{A}, \sigma) \vee \operatorname{ext}(\mathscr{A}, \sigma) \alpha$.

(2) If $\mathscr{A} \alpha=\mathscr{A}$, then

$$
\operatorname{ext}(\mathscr{A}, \sigma) \vee \operatorname{ext}(\mathscr{A}, \sigma) \alpha=\operatorname{ext}(\mathscr{A}, \sigma) \vee \operatorname{ext}(\mathscr{A}, \omega)
$$

Proof. (1). Set $m=\dot{\alpha}$ in (1.2). Then

$$
F_{\omega}(x)=\delta \partial_{\sigma}(\alpha x) \delta \partial_{\sigma}(x)^{-1}=\delta \partial_{\sigma}(\alpha) \delta \partial_{\sigma}(x) \delta \partial_{\sigma}(x)^{-1}=\delta \partial_{\sigma}(\alpha)
$$

since $\alpha \in \mathfrak{g}\left(\partial_{\sigma}\right)(\sec [2])$. 
Since al $(\delta \sigma) \subset \operatorname{ext}(\mathscr{A}, \sigma), \delta \sigma$ defines a continuous function on $|\operatorname{ext}(\mathscr{A}, \sigma)|$, the expression for $\delta \omega$ given in (1.2) shows that $\delta \omega$ defines a continuous function on

$$
|\operatorname{ext}(\mathscr{A}, \sigma) \vee \operatorname{ext}(\mathscr{A}, \sigma) \alpha| \text {. }
$$

(2). $\operatorname{By}(1), \mathcal{N}=\operatorname{ext}(\mathscr{A}, \sigma) \vee \operatorname{ext}(\mathscr{A}, \omega) \subset \operatorname{ext}(\mathscr{A}, \sigma) \vee \operatorname{ext}(\mathscr{A}, \sigma) \alpha=\mathscr{S}$. Since $\mathscr{A} \alpha=$ $\mathscr{A}, \mathscr{S}$ is a distal extension of $\mathscr{A}$ and so the proof may be completed by showing that

$$
N=\mathfrak{g}(\mathcal{N}) \subset S=\mathfrak{g}(\mathscr{S}) .
$$

To this end let

$$
\beta \in N=g(\operatorname{ext}(\mathscr{A}, \sigma)) \cap g(\operatorname{ext}(\mathscr{A}, \omega))
$$

Then

$$
\delta \sigma(\beta)=e=\delta \omega(\beta)
$$

whence.by (1.2)

$$
\begin{aligned}
e= & \delta \omega(\beta)=\delta \sigma(\beta)^{-1} \delta \sigma(\alpha)^{-1} \delta \sigma(\alpha \beta) \\
& =\delta \sigma(\alpha)^{-1} \delta \sigma(\alpha \beta) .
\end{aligned}
$$

Hence

$$
\delta \sigma(\alpha)=\delta \sigma(\alpha \beta)=\delta \alpha\left(\alpha \beta \alpha^{-1} \alpha\right)=\delta \sigma\left(\alpha \beta \alpha^{-1}\right) \delta \sigma(\alpha)
$$

(recall that $\mathscr{A} \alpha=\mathscr{A}$ implies that $\alpha A \alpha^{-1}=A$ whence $\alpha \beta \alpha^{-1} \in A \subset g(\sigma)$ ). This implies that

$$
\alpha \beta \alpha^{-1} \in \operatorname{ker} \delta \sigma
$$

and so

Consequently

$$
\beta \in \alpha^{-1} \operatorname{ker}(\delta \sigma) \alpha^{-1} \cap A=g(\operatorname{ext}(\mathcal{A}, \sigma) \alpha)
$$

$$
\beta \in S=g(\operatorname{ext}(\mathscr{A}, \sigma)) \cap g(\operatorname{ext}(\mathscr{A}, \sigma) \alpha) .
$$

(1.4) Corollary. Let

$$
\sigma \in Z(\mathscr{A}, K)
$$

and

$$
\mathscr{A} \alpha=\mathscr{A}\left(\alpha \in \mathfrak{g}\left(\partial_{\sigma}\right)\right)
$$

Then

$$
\bigvee\left\{\operatorname{ext}(\mathscr{A}, \sigma) \alpha \mid \alpha \in \mathfrak{g}\left(\partial_{\sigma}\right)\right\} \subset \operatorname{ext}(\mathscr{A}, \sigma) \bigvee \mathscr{E}
$$

(Here $\mathscr{E}$ is the set of all almost periodic functions on $\mathbb{Z}$.)

(1.5) Remarks. (1). Let $F$ be a $\tau$-closed subgroup of $G$ and $\mathcal{A}$ a $\mathbb{Z}$-sub-algebra of $a(u)$. Then it is natural to define $\mathcal{A}$ to be F-regular if $\mathscr{A} \alpha=\mathscr{A}(\alpha \in F)$ and $r_{F}(\mathcal{A})$, the $F$-regularizer of $\mathscr{A}$, as the supremum of $\{\mathscr{A} \alpha \mid \alpha \in F\}$. Then (1.4) states that

$$
r_{\mathrm{g}\left(\partial_{\sigma}\right)}(\operatorname{ext}(\mathscr{A}, \sigma)) \subset \operatorname{ext}(\mathscr{A}, \sigma) \vee \mathscr{E}
$$

if $\mathscr{A}$ is $g\left(\partial_{\sigma}\right)$-regular.

(2). Let $S=g(\operatorname{cxt}(\mathscr{A}, \sigma))$. Then (1.4) implies that

$$
S \cap E \subset \cap\left\{\alpha S \alpha^{-1} \mid \alpha \in \mathfrak{g}\left(\partial_{r}\right)\right\}
$$

when $\mathscr{A}$ is $g\left(\partial_{\sigma}\right)$-regular. (Here $E=g(\mathscr{E})$.) 
(1.6) Proposition. Let $\sigma \in Z(\mathscr{A}, K)$ and $\omega \in Z(M, K)$ witli $F_{\omega}=\delta \sigma$. Then

(1) $\partial_{\omega}=\sigma$.

(2) $\delta \omega\left(t^{k}\right)=\prod_{j=0}^{k-1} \delta \sigma\left(t^{j}\right)$.

(3) $\delta \omega(p t)=\delta \omega(p) \delta \sigma(p)(p \in \beta \mathbb{Z})$.

(4) $\delta \omega\left(p t^{k}\right)=\delta \omega(p) \prod_{j=0}^{k-1} \delta \sigma\left(p t^{j}\right)(p \in \beta \mathbb{Z})$.

(5) $\delta \omega\left(\beta t^{k}\right)=\delta \omega(\beta) \delta \sigma(\beta)^{k} \delta \omega\left(t^{k}\right)(\beta \in A=g(\mathcal{A}))$.

Proof. (1)

$$
(\delta \sigma)(m)=F_{\omega}(u)^{-1} F_{\omega}(m)=\left(\delta \partial_{\omega}\right)(u)^{-1}\left(\delta \partial_{\omega}\right)(m)=\left(\delta \partial_{\omega}\right)(m) \quad(m \in M) .
$$

Hence $\sigma=\partial_{\omega}$.

(2) $\delta \omega(t)=\omega(u, t)=F_{\omega}(u)=\delta \sigma(u)=e$ shows that

$$
\delta \omega\left(t^{k}\right)=\prod_{j=0}^{k-1} \delta \sigma\left(t^{j}\right) \quad \text { for } k=1 .
$$

Now assume that it holds for $1 \leq k \leq r$. Then

$$
\begin{aligned}
\delta \omega\left(t^{r+1}\right) & =\omega\left(u, t^{r+1}\right)=\omega\left(u, t^{r}\right) \omega\left(t^{r}, t\right) \\
& =\delta \omega\left(t^{r}\right) F_{\omega}\left(t^{r}\right)=\left(\prod_{j=0}^{r-1} \delta \omega\left(t^{j}\right)\right) \delta \omega\left(t^{r}\right) \\
& =\prod_{j=0}^{r} \delta \omega\left(t^{j}\right) .
\end{aligned}
$$

(3) Let $p \in \beta \mathbb{Z}$ and $t^{k_{1}} \rightarrow p$.Then $t^{k_{1}+1} \rightarrow p t$ and

$$
\begin{aligned}
\delta \omega(p t) & =\lim \delta \omega\left(t^{k_{i}+1}\right)=\left(\lim _{i} \prod_{i=0}^{k_{1}-1} \delta \sigma\left(t^{j}\right)\right) \lim _{i} \delta \sigma\left(t^{k_{i}}\right) \\
& =\lim _{i} \delta \omega\left(t^{k_{i}}\right) \lim _{i} \delta \sigma\left(t^{k_{i}}\right)=\delta \omega(p) \delta \sigma(p) .
\end{aligned}
$$

(4) This follows from (3) by induction on $k$.

(5) If $\beta \in A$ then $\delta \sigma(\beta x)=\delta \sigma(\beta) \delta \sigma(x)(x \in \beta \mathbb{Z})$. Hence

$$
\delta \omega\left(\beta t^{k}\right)=\delta \omega(\beta) \delta \sigma(\beta)^{k} \prod_{i=0}^{k-1} \delta \sigma\left(t^{j}\right)=\delta \omega(\beta) \delta \sigma(\beta)^{k} \delta \omega\left(t^{k}\right)
$$

(by (4) and (2)).

(1.7) Proposition. Let $\sigma \in Z(\mathscr{A}, \mathbb{K})$ be such that $\mathscr{S}=\operatorname{ext}(\mathscr{A}, \sigma)$ is weak-mixing and $\delta \sigma(A)=\mathbb{K}$. Then $\delta \omega(S)=\mathbb{K}$ where $\omega \in Z(M, \mathbb{K})$ with $F_{\omega}=\delta \sigma$ and $S=\mathrm{g}(\mathscr{S})$.

Proof. $\delta \omega(S)$ is a closed subgroup of $\mathbb{K}$ whence $\delta \omega(S)$ is finite or all of $\mathbb{K}$. If $\delta \omega(S)$ is finite then $\delta \omega^{n}(S)=e$ for some integer $n$. Since $\delta \sigma^{n}(A)=\mathbb{K}$ and $F_{\omega^{n}}=\delta \sigma^{n}$, it suffices to rule out the possibility that $\delta \omega(S)=e$.

Let $\mathscr{B}=\operatorname{ext}(\mathscr{S}, \omega)$, then $\delta \omega(S)=e$ implies that $\mathscr{B}=\mathscr{A}$ whence $\mathscr{B} \alpha=\mathscr{A} \alpha=\mathscr{A}=$ $\mathscr{B}(\alpha \in A)$. This is impossible since $\mathscr{B} \vee \mathscr{B} \alpha$ contains the eigenfunction $\delta p$ where $F_{p}(m)=\delta \sigma(\alpha) \quad(m \in M)$ by (1.3). (Recall that $\partial_{\omega}=\sigma$ and $A \subset g(\sigma)$.)

(1.8) Remarks. (1) The assumption $\delta \sigma(A)=\mathbb{K}$ implies that $|\mathscr{S}| \simeq \mathbb{K} \times|\mathcal{A}|$, and the conclusion $\delta \omega(S)=\mathbb{K}$ implies that

$$
|\operatorname{ext}(\mathscr{S}, \omega)|=\mathbb{K} \times \mathbb{K} \times|\mathfrak{A}| \text {. }
$$


We shall see later (corollary 1.11 ) that ext $(\mathscr{S}, \omega)$ is also weak-mixing.

(2) With the assumptions and notation of (1.7) let $\mathscr{B}=\operatorname{ext}(\mathscr{S}, \omega)$. (Observe that $F_{\omega}=\delta \sigma$ implies that $\omega \in Z(\mathscr{S}, \mathbb{K})$.) Then $r_{A}(\mathscr{B})=\mathscr{B} \vee \mathscr{E}$. To see this first observe that $S$ is a normal subgroup of $A$. This implies that $\mathscr{S}_{\alpha}=\mathscr{S}(\alpha \in A)$. Moreover $\partial_{\omega}=\sigma$ implies that $A \subset g\left(\partial_{\omega}\right)$. Hence by $(1.4) r_{A}(\mathscr{B}) \subset \mathscr{B} \vee \mathscr{E}$.

Now let $f$ be a character on $\mathbb{Z}$. Then $f=\delta p$ where $F_{\mathrm{p}}(m)=k$ for some $k \in \mathbb{K}$ and all $m \in M$. There exists $\alpha \in A$ with

$$
\delta \partial_{\omega}(\alpha)=\delta \sigma(\alpha)=k
$$

whence $f=\delta p \in r_{A}(\mathscr{S})$ by (1.3.). Since the characters generate

$$
\mathscr{E}, \mathscr{B} \vee \mathscr{E} \subset r_{A}(\mathscr{B}) \text {. }
$$

(3) Proposition 1.7 as well as theorem 1.10 below are true when $\mathbb{K}$ is replaced by a finite group of prime order.

(1.8) LeMmA. Let $\sigma \in Z^{1}(A, \mathbb{K})$ with $\delta \sigma(A)=\mathbb{K}, \varepsilon>0$ and $V$ a neighbourhood of u. Then there exists $p \in V \cap \vec{A} \subset M$ such that

$$
|\delta \sigma(p)-1| \leq \varepsilon \text { and }(\delta \sigma(p u))^{n} \neq 1 \text { if } n \neq 0 .
$$

(Notice that $p \in \bar{A}$ implies that $p u \in A$.)

Proof. Since $\delta \sigma(u)=1$ there exists a neighbourhood $W$ of $u$ with $\bar{W} \subset V$ and

$$
|\delta \sigma(x)-1| \leq \varepsilon \quad(x \in W) .
$$

By a now standard argument we may assume that

$$
\text { int }_{\tau} \text { cls }_{\tau}(W \cap A) \neq \varnothing .
$$

(See [3: 4.4].) Since $\delta \sigma:(A, \tau) \rightarrow \mathbb{K}$ is onto and open there exists

$$
\alpha \in \operatorname{cls}_{\tau}(W \cap A) \text { with } \delta \sigma(\alpha)^{n} \neq 1 \text { if } n \neq 0 .
$$

Let $\left(\alpha_{n}\right)$ be a net on $W \cap A$ with $\alpha_{n} \rightarrow \alpha$ and $\alpha_{n} \rightarrow p \in \beta \mathbb{Z}$. Then

$$
p \in \bar{W} \cap \bar{A} \subset V \cap \bar{A}, \quad|\delta \sigma(p)-1| \leq \varepsilon \quad \text { and } \quad \alpha_{n} \rightarrow p u .
$$

Hence $\delta \alpha(\alpha)=\delta \sigma(p u)$. The proof is completed.

(1.9) Proposition. Let $\sigma \in Z(\mathscr{A}, \mathbb{K})$ with $\delta \sigma(A)=\mathbb{K}, \omega \in Z(M, \mathbb{K})$ with $F_{\omega}=\delta \sigma$, and $\mathscr{S}=\operatorname{ext}(\mathscr{A}, \sigma)$. Then $\mathscr{B}=\operatorname{ext}(\mathscr{P}, \omega)$ is not an almost periodic extension of $\mathscr{A}$.

Proof. It will be convenient to identify $|\mathscr{B}|$ with a subset of $\mathbb{K} \times \mathbb{K} \times|\mathscr{A}|$. When this is done

$$
x \mid \mathscr{B}=(\delta \omega(x), \delta \sigma(x), x \mid \mathscr{A}) \quad(x \in M) .
$$

Let $\varepsilon>0$. We shall find $p, g \in \beta \mathbb{Z}$ such that $p|\mathscr{A}=u| \mathcal{A}, \quad|\delta \omega(p)-1| \leq \varepsilon, \quad|\delta \sigma(p)-1| \leq \varepsilon$ and $\left|\delta \omega(p g) \delta \omega(g)^{-1}-1\right| \geq \frac{1}{2}$. Thus $p \mid \mathscr{B}$ is close to $u|\mathscr{B}, p| \mathscr{A}=u \mid \mathscr{A}$, but $p g \mid \mathscr{B}$ is not close to $u g \mid \mathscr{B}$. Consequently $\mathscr{B}$ is not an almost periodic extension of $\mathscr{A}$.

To this end let $V$ be a neighbourhood of $u$ such that

$$
|\delta \omega(r)-1| \leq \varepsilon \quad(r \in V) .
$$


By (1.8) there exists $p \in V \cap \bar{A}$ with $|\delta \sigma(p)-1| \leq \varepsilon$ and $\delta \sigma(p u)^{n} \neq 1$ if $n \neq 0$. Since $p \in \bar{A}, p|\mathscr{A}=u| \mathscr{A}$.

Now choose $\lambda \in \mathbb{K}$ with $|\lambda \delta \omega(p u)-1|>\frac{1}{2}$. There exists a sequence of integers $k_{i}$ with $\delta \sigma(p u)^{k_{i}} \rightarrow \lambda$. Let $r \in \beta \mathbb{Z}$ be adherent to the sequence $t^{k_{i}}$. Then by (5) of (1.6)

$$
\delta \omega(p u r)=\delta \omega(p u) \lambda \delta \omega(r),
$$

whence $\left|\delta \omega(p u r) \delta \omega(r)^{-1}-1\right|>\frac{1}{2}$. Now set $u r=g$ and recall that $\delta \omega(r)=\delta \omega(u r)$. The proof is completed.

The following result is valid for any abelian group $T$.

(1.10) THEOREM. Let $\mathscr{S}$ be an almost periodic extension of $\mathscr{A}$ such that $S \triangleleft A$ and $A / S$ is a Lie group, and let $\mathscr{B}$ be an almost periodic extension of $\mathscr{S}$ such that $B \triangleleft S$ and $S / B \cong \mathbb{K}$. Then either

(i) $\mathscr{B}$ is an almost periodic extension of $\mathscr{A}$ or

(ii) $B \in S^{\perp \perp}$.

(Recall that

$$
\mathscr{R}^{\perp}=\{C \mid C \text { is a } \tau \text {-closed subgroup of } G \text { with } C R=G(R \in \mathscr{R})\}
$$

where $\mathscr{R}$ is a collection of $\tau$-closed subgroups of $G$.)

Proof. Assume that (i) does not hold and let $C \in S^{\perp}$; i.e. $C$ is a $\tau$-closed subgroup of $G$, with $C S=G$. Then

$$
C B \supset C S^{\prime} \supset G^{\prime}=E .
$$

Since $G / E$ is abelian, $C B$ is a normal subgroup of $G$.

Let $L=C B \cap S \supset E \cap S \supset E \cap A^{*}=A^{*}$. Hence $\mathscr{L}=\mathrm{a}(L) \cap \mathscr{A}^{*}$ is an almost periodic extension of $\mathscr{A}$. The exact sequences

$$
1 \rightarrow S / L \rightarrow A / L \rightarrow A / S \rightarrow 0 \text { and } S / B \rightarrow S / L \rightarrow 0
$$

show that $S / L$ is a circle or a point and that in either case $A / L$ is a Lie group.

Thus $\mathscr{A} \triangleleft \mathscr{L} \triangleleft \mathscr{B}$ and $L / B$ is a subgroup of the circle group $S / B$. Hence $L / B$ is finite or $L / B=S / B$. If $L / B$ were finite, $\mathscr{B}$ would be an almost periodic extension of $\mathscr{A}$ [7: 5.7], a possibility which has been ruled out. Therefore $L / B=S / B$ and so $L=S$. Consequently $S \subset C B$ and $G=C S \subset C B$. The proof is completed.

(1.11) Corollary. Let $\sigma \in Z(\mathscr{A}, \mathbb{K})$ with $\delta \sigma(A)=\mathbb{K}, \mathscr{S}=\operatorname{ext}(\mathscr{A}, \sigma)$ weak-mixing and $\omega \in Z(M, \mathbb{K})$ with $F_{\omega}=\delta \sigma$. Then $\mathscr{B}=\operatorname{ext}(\mathscr{S}, \omega)$ is weak-mixing and $\delta \omega(S)=\mathbb{K}$. Proof. Recall that when $T$ is abelian, a flow $(X, T)$ is weak-mixing if and only if $g(X) E=G$ (see [5:3.7 and 4]). By (1.9) and (1.10) $B \in S^{\perp \perp}$. Since $\mathscr{S}$ is weak-mixing, $S E=G$; i.e. $E \in S^{\perp}$. Hence $B E=G$ and $\mathscr{B}$ is weak-mixing. That $\delta \omega(S)=\mathbb{K}$ follows from (1.7).

We shall now use the results obtained to produce a weak-mixing flow with a non-weak-mixing quasi-factor.

(1.12) Notation. The following notation will be used for the remainder of this section: $\sigma \in Z(\mathscr{A}, \mathbb{K}), \mathscr{P}=\operatorname{ext}(\mathscr{A}, \sigma), \omega \in Z(M, \mathbb{K})$ with $F_{\omega}=\delta \sigma, \mathscr{B}=\operatorname{ext}(\mathscr{P}, \omega), \alpha \in$ $A$ with $\delta \sigma(\alpha) \neq 1,\langle\alpha\rangle$ the $\tau$-closed subgroup of $G$ generated by $\alpha$ and $\rho \in Z(M, \mathbb{K})$ with $F_{\rho}(m)=\delta \sigma(\alpha) \quad(m \in M)$. 
(1.13) Proposition. Let $\delta \omega(\beta)=1(\beta \in\langle\alpha\rangle)$. Then

(1) $\mathfrak{g}(\mathfrak{a}(\langle\alpha\rangle, \mathscr{B}))=\langle\alpha\rangle(\operatorname{ker} \delta \rho \cap B)$ and

(2) al $(\delta \rho) \subset \mathfrak{a}(\langle\alpha\rangle, \mathscr{B}))$.

Proof. (1) Set $\mathscr{L}=\mathfrak{a}(\langle\alpha\rangle, \mathscr{B})$ and $L=\mathrm{g}(\mathscr{L})$, and let $b \in \operatorname{ker} \delta \rho \cap B$. Then

$$
1=\delta \rho(b)=\delta \omega(b)^{-1} \delta \omega(\alpha)^{-1} \delta \omega(\alpha b)=\delta \omega(\alpha)^{-1} \delta \omega(\alpha b)
$$

(by (1.2)). Thus

$$
\delta \omega(\alpha)=\delta \omega(\alpha b)=\delta \omega\left(\alpha b \alpha^{-1} \alpha\right)=\delta \omega\left(\alpha b \alpha^{-1}\right) \delta \omega(\alpha) \text { since } \alpha b \alpha^{-1} \in S .
$$

Consequently $1=\delta \omega\left(\alpha b \alpha^{-1}\right)$ and $\alpha b \alpha^{-1} \in B=\operatorname{ker}(\delta \omega \mid S)$. Thus

$$
\alpha(\operatorname{ker} \delta \rho \cap B) \alpha^{-1} \subset \operatorname{ker} \delta \rho \cap B \text {. }
$$

Let $H=\{a \in\langle\alpha\rangle \mid a(\operatorname{ker} \delta \rho \cap B) \subset(\operatorname{ker} \delta \rho \cap B)\langle\alpha\rangle$. Then $H$ is a closed sub-semigroup of $\langle\alpha\rangle$. Hence $H$ is a closed subgroup of $\langle\alpha\rangle[1: 2.11]$. Since $\alpha \in H, H=\langle\alpha\rangle$. Consequently

$$
\langle\alpha\rangle(\operatorname{ker} \delta \rho \cap B) \subset(\operatorname{ker} \delta \rho \cap B)\langle\alpha\rangle
$$

and

$$
\begin{aligned}
(\operatorname{ker} \delta \rho \cap B)\langle\alpha\rangle & =(\langle\alpha\rangle \operatorname{ker} \delta \rho \cap B)^{-1} \subset((\operatorname{ker} \delta \rho \cap B)\langle\alpha\rangle)^{-1} \\
& =\langle\alpha\rangle(\operatorname{ker} \delta \rho \cap B) .
\end{aligned}
$$

Thus $\langle\alpha\rangle(\operatorname{ker} \delta \rho \cap B)$ is a $\tau$-closed subgroup of $G$.

Now $L$ is the largest $\tau$-closed subgroup of $G$ which contains $\langle\alpha\rangle$ and is contained in $\langle\alpha\rangle B$ [5: 3.1]. Hence $\langle\alpha\rangle(\operatorname{ker} \delta \rho \cap B) \subset L$.

Let $b \in L \cap B$. Then $\alpha b \in L=L^{-1} \subset(\langle\alpha\rangle B)^{-1}=B\langle\alpha\rangle$. Hence $\alpha b=r \beta$ for some $r \in B$ and $\beta \in\langle\alpha\rangle$. Then

$$
\delta \omega(\alpha b)=\delta \omega(r \beta)=\delta \omega(r) \delta \omega(\beta)=\delta \omega(\beta)=1 .
$$

Thus $\delta \rho(b)=\delta \omega(b)^{-1} \delta \omega(\alpha)^{-1} \delta \omega(\alpha b)=1$ and so $b \in \operatorname{ker} \delta \rho \cap B$.

Let $l \in L$. Then $l=k b$ for some $k \in\langle\alpha\rangle, b \in B$. Then

$$
b \in L \cap B \subset \operatorname{ker} \delta \rho \cap B
$$

and so $l \in\langle\alpha\rangle(\operatorname{ker} \delta \rho \cap B)$.

(2) $\delta \rho(\alpha)=\delta \omega(\alpha)^{-1} \delta \omega(\alpha)^{-1} \delta \omega\left(\alpha^{2}\right)=1$ shows that $\langle\alpha\rangle \subset$ ker $\delta \rho$. Hence

$$
L=\langle\alpha\rangle(\operatorname{ker} \delta \rho \cap B) \subset \operatorname{ker} \delta \rho=g(\text { al }(\delta \rho)) .
$$

Now al $(\delta \rho) \subset \mathscr{E}$, the algebra of almost periodic functions, implies that

$$
\mathfrak{a}(\langle\alpha\rangle, \mathscr{B}) \vee \text { al }(\delta \rho)
$$

is an almost periodic extension of $\mathfrak{a}(\langle\alpha\rangle, \mathscr{B})$. Since the groups of these flows are the same, the flows are equal. The proof is completed.

(1.14) Lemma. Let $\delta \omega(\alpha)=1=\delta \omega\left(\alpha^{2}\right)$. Then $\delta \omega\left(\alpha^{n}\right)=1$ for all integers $n$.

Proof. The formula

$$
\delta \rho(x)=\delta \omega(x)^{-1} \delta \omega(\alpha)^{-1} \delta \omega(\alpha x)=\delta \omega(x)^{-1} \delta \omega(\alpha x)
$$

shows that $\delta \rho(\alpha)=1$. Hence $\delta \rho(\langle\alpha\rangle)=1$ since $\delta \rho$ is a continuous homomorphism of $(G, \tau)$ into $K$. Lemma 1.14 now follows from (*) by induction. 
(1.15) LEMMA. $\delta \omega(\langle\alpha\rangle)=1$ if and only if $H(\langle\alpha\rangle, \tau) \subset B$ and $\delta \omega(\alpha)=1=\delta \omega\left(\alpha^{2}\right)$.

Proof. Let $\delta \omega((\alpha))=1$. Then of course $\delta \omega(\alpha)=1=\delta \omega\left(\alpha^{2}\right)$. Moreover $\alpha \in A$ implies that $\langle\alpha\rangle \subset A$. Hence

$$
H(\langle\alpha, \tau\rangle) \subset H(A, \tau) \subset A^{*} \subset S
$$

whence $H(\langle\alpha\rangle, \tau) \subset B=\operatorname{ker}(\delta \omega \mid S)$ since $\delta \omega(H(\langle\alpha\rangle, \tau)) \subset \delta \omega(\langle\alpha\rangle)=1$.

Now let $\delta \omega(\alpha)=1=\delta \omega\left(\alpha^{2}\right)$ and $H(\langle\alpha\rangle, \tau) \subset B$. Let $\beta \in\langle\alpha\rangle$. Choose a net $\left(\alpha^{N_{i}}\right)$ in $\langle\alpha\rangle$ with $\alpha^{N_{1}} \rightarrow{ }_{\tau} \beta$ and let $\alpha^{N_{i}} \rightarrow p \in M$. Then

$$
\delta \omega(p)=\lim \delta \omega\left(\alpha^{N_{i}}\right)=1
$$

by (1.14).

Moreover $\left(\alpha^{N_{1}}\right) \subset\langle\alpha\rangle \subset A$ implies that $p|\mathscr{A}=u| \mathscr{A}$ and $p u \in\langle\alpha\rangle$. Hence $p=p u$ on $\mathscr{B}(\mathscr{B}$ is a distal extension of $\mathscr{A})$ and $\delta \omega(p u)=\delta \omega(p)=1$. Also $\alpha^{N_{t}} \rightarrow_{\tau} p u$ shows that

$$
\beta(p u)^{-1} \in H(\langle\alpha\rangle, \tau) \subset B .
$$

Consequently $\delta \omega(\beta)=\delta \omega\left(\beta(p u)^{-1} p u\right)=\delta \omega\left(\beta(p u)^{-1}\right) \delta \omega(p u)=1$.

(1.16) $A$ construction. Let $\mathscr{A}$ be a weak-mixing metric flow and $\sigma \in Z(\mathscr{A}, \mathbb{K})$ such that $\mathscr{P}=\operatorname{ext}(\mathscr{A}, \sigma)$ is weak-mixing and $\delta \sigma(A)=\mathbb{K}$. (Such exist, see [6].) Set $\mathscr{B}=\operatorname{ext}(\mathscr{P}, \omega)$ where $F_{\omega}=\delta \sigma$. Then by $(1.11) \mathscr{B}$ is weak-mixing and $\delta \omega(B)=\mathbb{K}$. Hence $|\mathscr{B}|$ may be identified with the flow $(\mathbb{K} \times \mathbb{K} \times|\mathscr{A}|, t)$ where

$$
(k, l, x) t=(\delta \sigma(m t), \delta \omega(p t), x t),
$$

$(k, l \in \mathbb{K}, x \in|\mathscr{A}|$ and $m, p \in M$ with $\delta \sigma(m)=k, \delta \omega(p)=l)$.

Let $\lambda \in \mathbb{K}$. Then the flow $\left(\mathbb{K}, R_{\lambda}\right)$ is equicontinuous and so is disjoint from $\mathscr{B}$. Hence there exists a sequence $\left(N_{i}\right)$ such that

$$
\left(1,1, x_{0}\right) t^{N_{1}} \rightarrow\left(1, \lambda, x_{0}\right) \text { and } \lambda^{N_{1}} \rightarrow 1 \text {. }
$$

$\left(\right.$ Here $\left.x_{0}=u \mid A_{.}\right)$

Let $p \in \beta \mathbb{Z}$ be a limit point of the sequence $\left(t^{N_{i}}\right)$ and set $\alpha=u p u \in G$.

(1.16.1) $\delta \omega\left(\alpha^{k}\right)=1$ for all integers $k$.

Proof. Since $\left(1,1, x_{0}\right) t^{N_{t}} \rightarrow\left(1, \lambda, x_{0}\right),\left(1,1, x_{0}\right) p=\left(1, \lambda, x_{0}\right)$. Also

$$
\left(1,1, x_{0}\right) p=\left(1,1, x_{0}\right) u p=\left(\delta \omega(u p), \delta \sigma(u p), x_{0} u p\right) \text {. }
$$

Hence $\delta \omega(u p)=1, \delta \sigma(u p)=\lambda$ and $u p=u$ on $\mathscr{A}$. Thus $\alpha=u p u=u$ on $\mathscr{A}$; i.e. $\alpha \in A$. Since $\mathscr{S}$ and $\mathscr{B}$ are both distal extensions of $\mathscr{A}$ and $u p=u p u$ on $\mathscr{A}, \alpha=u p u=u p$ on $\mathscr{S}$ and $\mathscr{B}$. Consequently $\delta \omega(\alpha)=\delta \omega(u p)=1$ and $\delta \sigma(\alpha)=\lambda$.

Moreover by (5) of (1.6)

$$
\delta \omega\left(\alpha t^{N_{i}}\right)=\delta \omega(\alpha) \delta \sigma(\alpha)^{N_{i}} \delta \omega\left(t^{N_{1}}\right)=\lambda^{N_{1}} \delta \omega\left(t^{N_{1}}\right)
$$

from which it follows that

$$
\delta \omega(\alpha p)=\delta \omega(p)=\delta \omega(\alpha)=1 .
$$

Since $\alpha p u=p u=p=\alpha p$ on $\mathscr{A}, \alpha^{2}=\alpha p u=\alpha p$ on $\mathscr{B}$. Consequently $\delta \omega\left(\alpha^{2}\right)=$ $\delta \omega(\alpha p)=1$ and (1.16.1) follows from (1.14).

(1.16.2) Let $\lambda^{k}=1$. Then $H(\langle\alpha\rangle, \tau) \subset B$. 
Proof. $\delta \sigma\left(\alpha^{k}\right)=\delta \sigma(\alpha)^{k}=\lambda^{k}=1$ implies that $\alpha^{k} \in S$. By (1.16.1), $\delta \omega\left(\alpha^{k}\right)=1$. Hence $\alpha^{k} \in B=\operatorname{ker}(\delta \omega \mid S)$.

Consequently $\langle\alpha\rangle \cap B$ has finite index in $\langle\alpha\rangle$ whence

$$
H(\langle\alpha\rangle, \tau) \subset\langle\alpha\rangle \cap B \subset B .
$$

(1.16.3) Let $\lambda^{k}=1$ with $\lambda \neq 1, k \neq 0$. Then $\mathrm{a}(\langle\alpha\rangle, \mathscr{B})$ is a non-weak-mixing quasifactor of the weak-mixing flow, $\mathscr{B}$.

Proof. This follows from (1.16.2), (1.15), and (1.13).

\section{Section 2}

(2.1) Definition. Let $\sigma \in Z(\mathscr{A}, K)$. Then the sequence built on $(\mathscr{A}, \sigma)$ is the sequence $\left(\mathscr{A}_{n}, \sigma_{n}\right)(n \geq 0)$ defined inductively as follows: $\mathscr{A}_{0}=\mathscr{A}_{1}, \sigma_{0}=\sigma, \mathscr{A}_{n+1}=\operatorname{ext}\left(\mathscr{A}_{n}, \sigma_{n}\right)$ and $\sigma_{n+1} \in Z\left(\mathscr{A}_{n}, K\right)$ with $F_{\sigma_{n+1}}=\delta \sigma_{n}$. Thus $\partial_{\sigma_{n+1}}=\sigma_{n}$.

In $\S 1$ we were concerned with the first two or three terms of the sequence built on $(\mathscr{A}, \sigma)$. In particular (1.3) dealt with $\mathscr{A}_{1} \vee \mathscr{A}_{1} \alpha$ for $\alpha \in \mathrm{g}\left(\partial_{\sigma}\right)$. Here we shall consider $\mathscr{A}_{n} \vee \mathscr{A}_{n} \alpha$ for $\alpha \in A=\mathrm{g}(\mathscr{A})$.

(2.2) Notation. For most of this section we shall be dealing with a fixed flow $\mathscr{A}$, $\sigma \in Z(\mathscr{A}, K)$, and $\alpha \in A$. Let $\left(\mathscr{A}_{n}, \sigma_{n}\right)$ be the sequence built on $(\mathscr{A}, \sigma)$ and

$$
k_{n}=\delta \sigma_{n}(\alpha) \in K \quad(n \geq 0) .
$$

Then $\left(\mathscr{S}_{i}^{n}, \rho_{i}^{n}\right)_{i \geq 0}$ will denote the sequence built on $\left(\mathbb{R}, \rho^{n}\right)(n \geq 0)$ where

$$
\rho^{n} \in Z(M, K) \text { with } F_{\rho^{n}}(m)=k_{n} \quad(m \in M) .
$$

The various flows and cocycles depend of course on $\alpha$ but this dependence has been suppressed for 'notational convenience'.

(2.3) Propositron. For all positive integers $n$,

$$
\prod_{i=0}^{n} \delta \rho_{n-1}^{i}(x)=\delta \sigma_{n+1}(x)^{-1} \delta \sigma_{n+1}(\alpha)^{-1} \delta \sigma_{n+1}(\alpha x) \quad(x \in M) .
$$

Proof. The case $n=0$ is just proposition 1.2. Now assume that

$$
\prod_{i=0}^{n-1} \delta \rho_{n-1-i}^{i}(x)=\delta \sigma_{n}(x)^{-1} \delta \sigma_{n}(\alpha)^{-1} \delta \sigma_{n}(\alpha x) \quad(x \in M) .
$$

Then $\mathscr{A}_{n+1}=\operatorname{ext}\left(\mathscr{A}_{n}, \sigma_{n}\right)$ and $\partial_{\sigma_{n+1}}=\sigma_{n}$. By $(1.2)$ if $\gamma \in Z(M, K)$ with

$$
\delta \gamma(x)=\delta \sigma_{n+1}(x)^{-1} \delta \sigma_{n+1}(\alpha)^{-1} \delta \sigma_{n+1}(\alpha x)
$$

then $F_{\gamma}(x)=\delta \sigma_{n}(\alpha x) \delta \sigma_{n}(x)^{-1} \quad(x \in M)$. Thus

$$
\begin{aligned}
F_{y}(x) & =\delta \sigma_{n}(\alpha) \prod_{i=0}^{n-1} \delta \rho_{n-1-i}^{i}(x) \\
& =F_{\rho \hat{0}}(x) \prod_{i=0}^{n-1} F_{\rho_{n-1}^{\prime}}(x)=\prod_{i=0}^{n} F_{\rho_{n-1}^{\prime}}(x) .
\end{aligned}
$$

Consequently $\gamma=\prod_{i=0}^{n} \rho_{n-1}^{l}$ and $\delta \gamma=\prod_{i=0}^{n} \delta \rho_{n-1}^{l}$ (recall that $K$ is abelian). The proof is completed. 
(2.4) Remarks. We should now like to use the cocycles $\gamma_{n}=\prod_{i=0}^{n} \rho_{n-i}^{i}$ to build a sequence of flows $\left(\mathscr{R}_{n}\right)$. To this end observe that

$$
\delta \gamma_{n}=\prod_{i=0}^{n} \delta \rho_{n-i}^{l}=\prod_{i=0}^{n} F_{\rho_{n+i-1}^{l}} \text { and } F_{\gamma_{n+1}}=\prod_{i=0}^{n+1} F_{\rho_{n+1-i}^{\prime}}
$$

whence

$$
F_{\gamma_{n+1}}=\left(\delta \gamma_{n}\right) F_{\rho_{0}^{n+1}} .
$$

Now set $\mathscr{R}_{0}=\mathbb{R}$. Since $\gamma_{0}$ is the constant cocycle $\rho_{0}^{0}, \mathscr{R}_{1}=\operatorname{ext}\left(\mathscr{R}_{0}, \gamma_{0}\right)$ is an almost periodic extension of $\mathscr{R}_{0}$.

Assume that $\gamma_{n}$ is a cocycle on $\mathscr{R}_{n}$ to $K$ and set $\mathscr{R}_{n+1}=\operatorname{ext}\left(\mathscr{R}_{n}, \gamma_{n}\right)$. Then $\delta \gamma_{n}$ defines a continuousfunction on $\left|\mathscr{R}_{n+1}\right|$ to $K$. Since $F_{\rho_{0}^{n+1}}$ is constant, $F_{\gamma_{n+1}}=\left(\delta \gamma_{n}\right) F_{\rho_{0}^{n+1}}$ defines a continuous function on $\left|\mathscr{R}_{n+1}\right|$ to $K$. Hence $\gamma_{n+1}$ is a cocycle on $\mathscr{R}_{n+1}$ to $K$ and the sequence $\left(\mathscr{R}_{n}\right)$ is well defined, where $\mathscr{R}_{k+1}=\operatorname{ext}\left(\mathscr{R}_{k}, \gamma_{k}\right)$.

(2.5) Proposition. For all integers $n \geq 1, \mathscr{A}_{n} \vee \mathscr{A}_{n} \alpha=\mathscr{A}_{n} \vee \mathscr{R}_{n-1}$.

Proof. When $n=1, \mathscr{R}_{0}=\mathbb{B}$ and $\mathscr{A}_{1} \vee \mathscr{R}_{0}=\mathscr{A}_{1}$. Moreover $\mathscr{A}_{1} \vee \mathscr{A}_{1} \alpha=\mathscr{A}_{1}$ since $\alpha \in$ $A=A_{0}$ and $A_{1} \triangleleft A_{0}$.

The case $n=2$ is just (1.3).

Assume that $\mathscr{A}_{n} \vee \mathscr{A}_{n} \alpha=\mathscr{A}_{n} \vee \mathscr{R}_{n-1}$. Since $\mathscr{A}_{n+1}$ and $\mathscr{R}_{n}$ are distal extensions of $\mathscr{A}_{n}$ and $\mathscr{R}_{n-1}$ respectively $\mathscr{A}_{n+1} \vee \mathscr{A}_{n+1} \alpha$ and $\mathscr{A}_{n+1} \vee \mathscr{R}_{n}$ are both distal extensions of the flow

$$
\mathscr{A}_{n} \vee \mathscr{A}_{n} \alpha=\mathscr{A}_{n} \vee \mathscr{R}_{n-1} .
$$

It thus suffices to show that their groups $A_{n+1} \cap \alpha^{-1} A_{n+1} \alpha$ and $A_{n+1} \cap R_{n}$ are equal.

Let $\beta \in A_{n+1} \cap \alpha^{-1} A_{n+1} \alpha \subset A_{n} \cap \alpha^{-1} A_{n} \alpha=A_{n} \cap R_{n-1}$. Then

$$
\delta \gamma_{n-1}(\beta)=\prod_{i=0}^{n-1} \delta \rho_{n-1-i}^{i}(\beta)=\delta \sigma_{n}(\beta)^{-1} \delta \sigma_{n}(\alpha)^{-1} \delta \sigma_{n}(\alpha \beta)
$$

and $\beta \in A_{n+1} \cap \alpha^{-1} A_{n+1} \alpha$ implies that $\delta \sigma_{n}(\beta)=e$ and

$$
\delta \sigma_{n}(\alpha \beta)=\delta \sigma_{n}\left(\alpha \beta \alpha^{-1} \alpha\right)=\delta \sigma_{n}\left(\alpha \beta \alpha^{-1}\right) \delta \sigma_{n}(\alpha) .
$$

Thus $\delta \gamma_{n-1}(\beta)=e$; which together with the fact that $\beta \in R_{n-1}$ implies that $\beta \in R_{n}$. Consequently

$$
A_{n+1} \cap \alpha^{-1} A_{n+1} \alpha \subset A_{n+1} \cap R_{n} .
$$

Now let $\beta \in A_{n+1} \cap R_{n}$. Then $e=\delta \gamma_{n-1}(\beta)=\delta \sigma_{n}(\beta)^{-1} \delta \sigma_{n}(\alpha)^{-1} \delta \sigma_{n}(\alpha \beta)$ whence $\delta \sigma_{n}(\alpha \beta)=\delta \sigma_{n}(\alpha) \delta \sigma_{n}(\beta)$. On the other hand

$$
\delta \sigma_{n}(\alpha \beta)=\delta \sigma_{n}\left(\alpha \beta \alpha^{-1} \alpha\right)=\delta \sigma_{n}\left(\alpha \beta \alpha^{-1}\right) \delta \sigma_{n}(\alpha) .
$$

(Recall $\beta \in A_{n+1} \cap R_{n} \subset A_{n} \cap R_{n-1}=A_{n} \cap \alpha^{-1} A_{n} \alpha$.) Hence $\delta \sigma_{n}\left(\alpha \beta \alpha^{-1}\right)=e$ and so $\beta \in \alpha^{-1} A_{n+1} \alpha$. The proof is completed.

(2.6) Remarks. We should now like to consider the $A$-regularizer, $r_{A}\left(\mathscr{A}_{n}\right)$ of $\mathscr{A}_{n}$. To this end we introduce the following notation. Let $\kappa \in K$. Then $\left(\mathscr{S}_{n}^{\alpha} \mid n=0, \ldots\right)$ will denote the sequence built on $(\mathbb{R}, \eta)$ where $\eta$ is the cocycle on $M$ to $K$ with $F_{n}(m)=\kappa \quad(m \in M)$. We shall also denote by $\mathscr{R}_{n}(\alpha)$ the flow previously denoted by $\mathscr{R}_{n}$ in order to indicate its dependence on $\alpha \in A$. 
Let $\mathscr{E}_{n}=\bigvee_{\kappa \in K} \mathscr{S}_{n}^{\kappa}$. Then we shall show that $\delta \sigma_{n}\left(A_{n}\right)=K$ for all $n$ implies that $r_{A}\left(\mathscr{A}_{n}\right)=\mathscr{A}_{n} \vee \mathscr{E}_{n-1}$ for all $n$.

(2.7) Lemma. Let $\mathscr{L}, \mathscr{K}$, and $\mathcal{N}$ be minimal flows such that $\mathscr{K}$ is a distal extension of $\mathscr{L}, \mathscr{L} \subset \mathcal{N}$, and $N \subset K$. Then $\mathscr{K} \subset \mathcal{N}$.

Proof. By [1: 13.11], $\mathrm{g}\left(\mathscr{L}^{*} \cap \mathcal{N}\right)=L^{*} N$ and $\mathrm{g}\left(\mathscr{L}^{*} \cap \mathscr{K}\right)=L^{*} K \supset L^{*} N$. Consequently

$$
\mathscr{K}=\mathscr{L}^{*} \cap \mathscr{K} \subset \mathscr{L}^{*} \cap \mathcal{N} \subset \mathcal{N} \text {. }
$$

(2.8) LemmA. For all integers $n \geq 1$ and all $\alpha \in A, \mathscr{R}_{n}(\alpha) \subset \mathscr{E}_{n}$.

Proof. Since $\mathscr{R}_{1}(\alpha)=\mathscr{S}_{1}^{F \gamma_{0}(\alpha)}, \mathscr{R}_{1}(\alpha) \subset \mathscr{C}_{1}$. Assume $\mathscr{R}_{n}(\alpha) \subset \mathscr{E}_{n}$. Then

Since

$$
\mathscr{R}_{n+1}(\alpha)=\operatorname{ext}\left(\mathscr{R}_{n}(\alpha), \gamma_{n}\right) \quad \text { with } F_{\gamma_{n}}=\prod_{i=0}^{n} F_{\rho_{n-1}^{\prime}} .
$$

$$
\mathscr{P}_{0}^{\kappa_{n+1}} \vee \cdots \vee \mathscr{S}_{n+1}^{\kappa_{0}} \subset \mathscr{E}_{n+1} \quad\left(\kappa_{l}=F_{\mathrm{\rho d}}(\alpha)\right), \quad \delta \gamma_{n}(\beta)=e \quad\left(\beta \in E_{n+1}\right) .
$$

Lemma 2.8 now follows from (2.7).

(2.9) LeMMA. Let $\delta \sigma_{i}\left(A_{i}\right)=K, \kappa_{i} \in K(1 \leq i \leq n)$. Then there exists $\alpha \in A$ with

$$
\delta \sigma_{i}(\alpha)=\kappa_{i} \quad(1 \leq i \leq n) \text {. }
$$

Proof. The hypothesis implies that $\mathscr{A}_{n+2}$ may be identified with a flow whose underlying phase space is $K^{n} \times|\mathscr{A}|$ and such that

$$
\left(e, \ldots, e, x_{0}\right) p=\left(\delta \sigma_{n}(p), \ldots, \delta \sigma(p), x_{0} p\right) \quad(p \in M) .
$$

Hence there exists $p \in M$ with

$$
\delta \sigma_{i}(p)=\kappa_{l} \quad(1 \leq i \leq n) \text { and } x_{0} p=x_{0} ;
$$

i.e. $p=u$ on $\mathscr{A}$. Then

$$
\alpha=u p u \in A \text { and } \delta \sigma_{i}(\alpha)=\kappa_{i} \quad(1 \leq i \leq n) .
$$

(2.10) LeMmA. Let $\delta \sigma_{l}\left(A_{l}\right)=K$ for all $i$. Then, for all $n$,

$$
\mathscr{E}_{n} \subset v\left\{\mathscr{R}_{n}(\alpha) \mid \alpha \in A\right\} .
$$

Proof. Since $\mathscr{S}_{1}^{\delta \sigma_{0}(\alpha)}=\mathscr{R}_{1}(\alpha)$ and $\delta \sigma_{0}(A)=K$,

$$
\mathscr{E}_{1} \subset \vee\left\{\mathscr{R}_{1}(\alpha) \mid \alpha \in A\right\} \text {. }
$$

Now assume that

$$
\mathscr{E}_{n} \subset \vee\left\{\mathscr{R}_{n}(\alpha) \mid \alpha \in A\right\}
$$

and let $\kappa \in K$. Choose $\alpha \in A$ with $\delta \sigma_{0}(\alpha)=\kappa$ and $\delta \sigma_{i}(\alpha)=e(1 \leq i \leq n)$.

In this case the cocycles $\rho^{k}$ are trivial for $1 \leq k \leq n$ and $\rho^{0}$ is just the cocycle with $F_{\rho} 0 \equiv \kappa$. Consequently $\gamma_{n}=\rho_{n}^{0}$. Since

$$
\begin{gathered}
\mathscr{R}_{n+1}(\alpha)=\operatorname{ext}\left(\mathscr{R}_{n}(\alpha), \gamma_{n}\right) \quad \text { and } \mathscr{S}_{n+1}^{\alpha}=\operatorname{ext}\left(\mathscr{S}_{n}^{\alpha}, \rho_{n}^{0}\right), \\
\delta \rho_{n}^{0}(\beta)=e \quad\left(\beta \in R_{n+1}(\alpha)=g\left(\mathscr{R}_{n+1}(\alpha)\right)\right) .
\end{gathered}
$$

Hence $g\left(\vee\left\{\mathscr{R}_{n+1}(\beta) \mid \beta \in A\right\}\right) \subset g\left(\mathscr{S}_{n+1}^{\alpha}\right)$ and so

$$
\mathscr{S}_{n+1}^{\alpha} \subset \vee\left\{\mathscr{R}_{n+1}(\beta) \mid \beta \in A\right\}
$$

by (2.7). Since $\kappa$ was arbitrary, $\mathscr{E}_{n+1} \subset \vee\left\{\mathscr{R}_{n+1}(\beta) \mid \beta \in A\right\}$. 
(2.11) Proposition. Let $\delta \sigma_{n}\left(A_{n}\right)=K$ for all $n$. Then $r_{A}\left(\mathscr{A}_{n}\right)=\mathscr{A}_{n} \vee \mathscr{E}_{n-1}$ for all $n$.

Proof. Let $\alpha \in A$. Then $\mathscr{A}_{n} \vee \mathscr{A}_{n} \alpha=\mathscr{A}_{n} \vee \mathscr{R}_{n-1}(\alpha) \subset \mathscr{A}_{n} \vee \mathscr{E}_{n-1}$ by (2.5) and (2.8).

Hence

$$
r_{A}\left(\mathscr{A}_{n}\right)=\vee\left\{\mathscr{A}_{n} \alpha \mid \alpha \in A\right\} \subset \mathscr{A}_{n} \vee \mathscr{E}_{n-1}
$$

On the other hand

$$
\mathscr{R}_{n-1}(\alpha) \subset \mathscr{A}_{n} \vee \mathscr{A}_{n} \alpha \subset r_{A}\left(\mathscr{A}_{n}\right) \quad(\alpha \in A)
$$

implies that $\mathscr{A}_{n} \vee \mathscr{E}_{n-1} \subset r_{A}\left(\mathscr{A}_{n}\right)$ by (2.10).

(2.12) Remark. Corollary 1.11 shows that the condition that $\delta \sigma_{n}\left(A_{n}\right)=K$ for all $n$ is satisfied in the case when $\mathscr{A}$ is weak-mixing, $K=\mathbb{K}$, and $\delta \sigma(A)=K$.

R.E.'s research was supported by NSF Grant no. 7800403 A02.

\section{REFERENCES}

[1] R. Ellis. Lectures in Topological Dynamics. Benjamin: New York, 1969.

[2] R. Ellis. Cocycles in topological dynamics. Topology 17 (1978), 111-130.

[3] R. Ellis. The Veech structure theorem, Trans. Amer. Math. Soc. 186 (1973), 203-218.

[4] R. Ellis \& S. Glasner. Pure weak mixing. Trans. Amer. Math. Soc. 243 (1978), 135-146.

[5] R. Ellis, S. Glasner \& L. Shapiro. Algebraic equivalents of flow disjointness. Illinois J. Math. 20 (1976), 354-360.

[6] R. Peleg. Some extensions of weakly mixing flows. Israel J. Math. 9 (1971), 330-336.

[7] $M$. Rees. On the structure of minimal distal transformation groups with topological manifolds as phase spaces, Maths. Inst. Univ. of Warwick, Coventry (1977). 